\title{
Development of a Remote Embedded System Controlled by a Mobile Device
}

\author{
SungilYoo ${ }^{1}$, Hyeok $\mathrm{Oh}^{2}$, Eun-Ji Kim ${ }^{2}$ and Dongik $\mathrm{Oh}^{2}$ \\ ${ }^{1}$ Graduate School of Medical Sciences, \\ ${ }^{2}$ Department of Medical IT Engineering \\ SoonChunHyang University, Asan, Korea \\ sgyoo@sch.ac.kr,oh4851@gmail.com,v_eunji_v@naver.com, \\ dohdoh@sch.ac.kr
}

\begin{abstract}
Remotely controlled Home Automation is popular these days, but it is usually applicable to recent appliances only. In order to utilize old devices in the automation we have developed a model that integrates conventional electriclelectronic devices into the automation. We have named the modelWhaut (Wireless Home AUTomation), in which a mobile terminal is used to trigger the control through Weband an embedded unit is used to control the target appliances. We have demonstrated usefulness of theWhaut model by implementing a mobile terminal basedsystem for controlling a conventional air-conditioner.
\end{abstract}

Keywords: home automation, remote control, mobile terminal, electronic device, web application, embedded system

\section{Introduction}

Wide adoption of a mobile handset enables us to experience computing power anywhere anytime. We are living in the ubiquitous computing era that Mark Weiser has foreseen a few decades ago [1]. Connected to numerous computing devices through network, we can experience many new novel applications that make our lives more comfortable and exciting.Home Automation is one of such areas where we may experience new ways to deal with our daily activities [2]. By combining in-house applianceswith Web technology, we may provide many new novelremote applications. If the mobile handset is used to trigger and monitor the in-house electric/electronic devices, user satisfaction would be further magnified.

This kind of remotely controlled Home Automation system is already in the market with the functionality imbedded into the devices by the manufacturer. However, such systems are proprietarily structured so that combining them with other devices is usually impossible. Also the price of the device with suchfunctionalities is way too expensive compare to the ones without. On these observations we have sought a way to utilize conventional electric/electronic devices in the Web controlled Home Automation. We have built a model for the integration and named the modelWhaut (Web based Home AUTomation). In this paper we describe the structure of the Whaut model and report on the application of the model to implement a system that controls an air-conditionerunit via a mobile app 


\section{Whaut Model}

Whaut model consists of a mobile client, a host station, an embedded module, and electric/electronic devices to be controlled. Software modules installed onto each unit play important roles to bring up the remote automation possible.Many different software technologies may be used to implement the Whaut model, but in this study we have used widely adapted and efficient technologies for the easy and stableimplementation of the system[3].

Overall structure of Whaut is shown in Figure 1. The mobile device uses its web browser to connect to the host PC which acts as a web server. JQueryMobile integrated in HTML5 page is used to create UX of the model and JSP is used to bring up server side logic. A servlet that catches the web request communicates with a daemon (AVRdaemon) that monitors the embedded device.The embedded device has two major functionalities. One is to monitor the sensors connected to the embedded system so that intelligent decisions needed by the system can be made. The second functionality is to control the electronic devices connected to the embedded module. Of course the electronic device does not necessary to be wired to the embedded module.

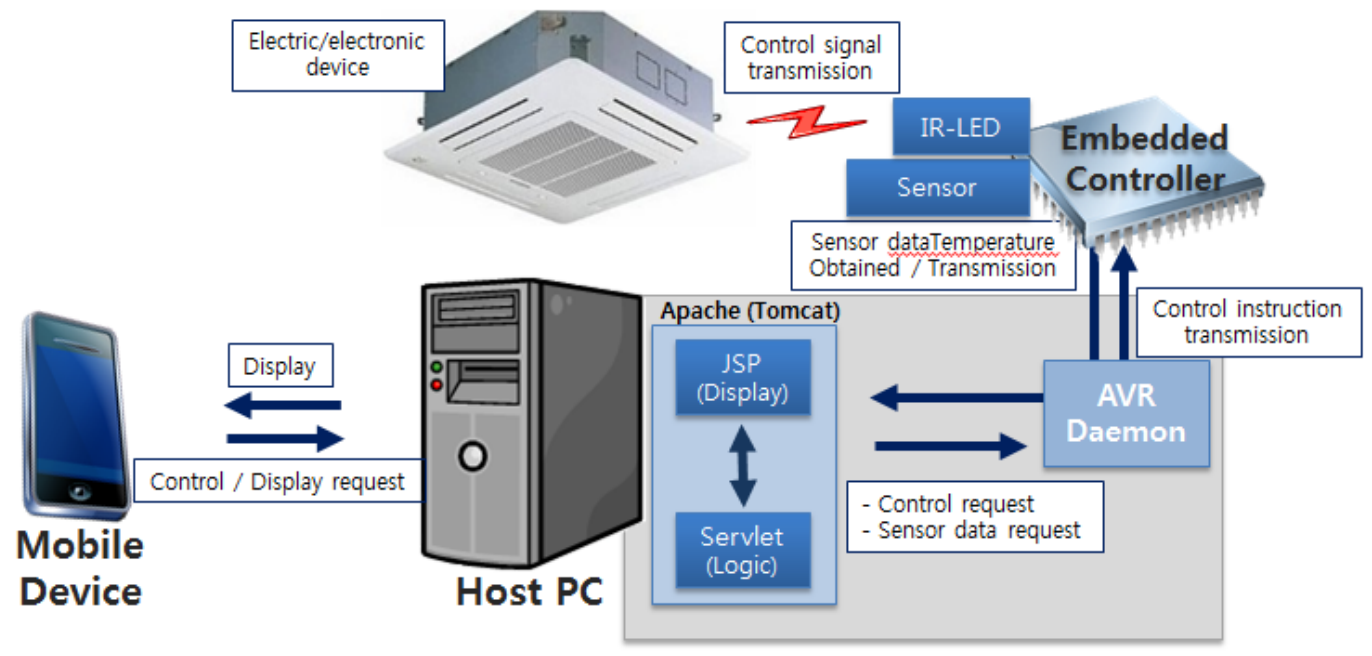

Figure 1. Overall Structure of the Whaut Model

Whaut model is server/client model that is flexible enough to be applied to various remote Home Automationsystems.

\section{Application of Whaut modelfor the Air-Conditioner Control}

Many recent appliances come with Home Automation capabilities, but not the old ones. In order to utilize old models in the automation, we developed the Whautmodel and used it to implement an air-conditioner control system over the web using a mobile phone. This demonstrates that Whaut model is applicable to various remote control systems, such as airconditioners, heaters, and any device that can be controlled by anIR (Infrared Ray) remote control. The overall structure of the air-conditioner controlling systembased on the Whaut model is shown in Figure 2. 


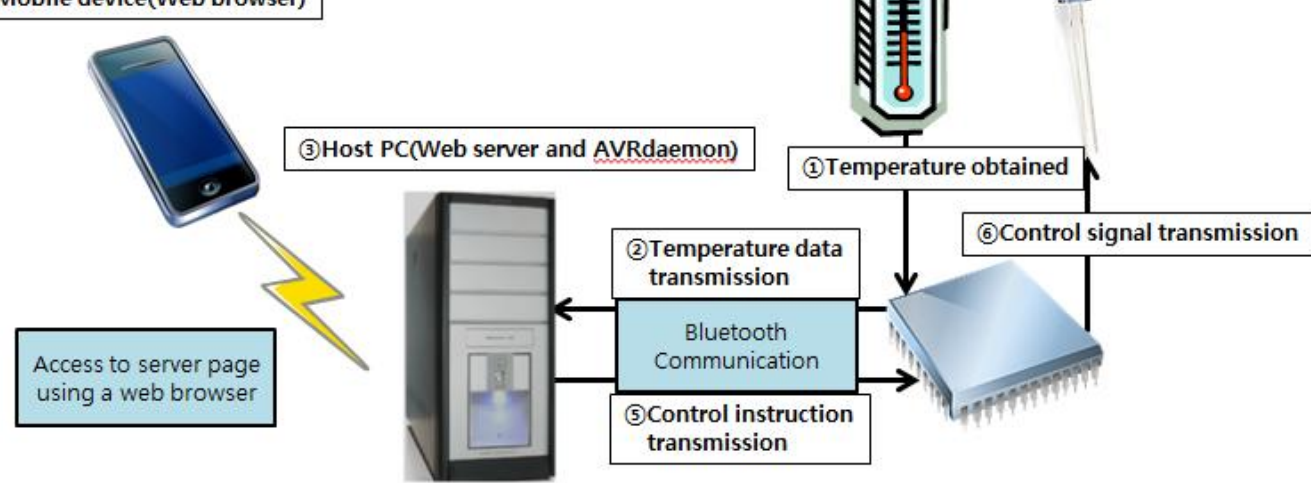

Figure 2. Air-Conditioner Controlling System based on the Whaut Model

Operational scenario of the system is as follows: (1) An embedded module equipped with a thermometer gets the room temperature. (2) The temperature reading is sent to the host PC via Bluetooth communication. (3)AVRdaemon on the host PC monitors the embedded unit and stores the temperature setting that the user requests. (4)Users may retrieve the current temperature through the web server. Users can also send control command to the embedded module using the mobile terminal. (5) The server relay the information gotten from the user to the AVRdaemon and it is then send to the embedded device. (6) The embedded device preparesa control command for the airconditioner and sends it to the air conditioner using IR signal.

\section{Infrared Ray (IR) Communication}

To control the air-conditioner unit, we used IR communication used by the remote controller of the appliance. To do so we needed to analyze the signal pattern of the IR communication system. We assembled a microcontroller with an IR receiver and programmed a counter that is incremented at 100us interval. An interrupt takes place when the receiver gets an IR signal. We counted time between the rising-edge and falling-edge of the signal, and found out how long we need to turn the signal on and off to control the unit.

In IR systems, use of NEC format which consists of a Lead Code followed by 32bit Data Code is common [4]. However, the air-conditioner used in this project has a different format. Its Data Code is consists of 56bits. Expression of Lead Code and the Data Code of the airconditioner is summarized in Figure 3. For the Lead Code, we need to turn the IR LED on for $3 \mathrm{~ms}$ and turn it off for 9ms. ' 0 ' bit in the Data Code is expressed by $0.56 \mathrm{~ms}$ LED on and $0.565 \mathrm{~ms}$ LED off. ' 1 'bit is expressed by $0.56 \mathrm{~ms}$ LED on and $1.69 \mathrm{~ms}$ LED off. 
With the information gathered, we prepared an embedded unit to emit appropriate signal pattern (IR LED on/off patterns) according to the request received through AVRdaemon.
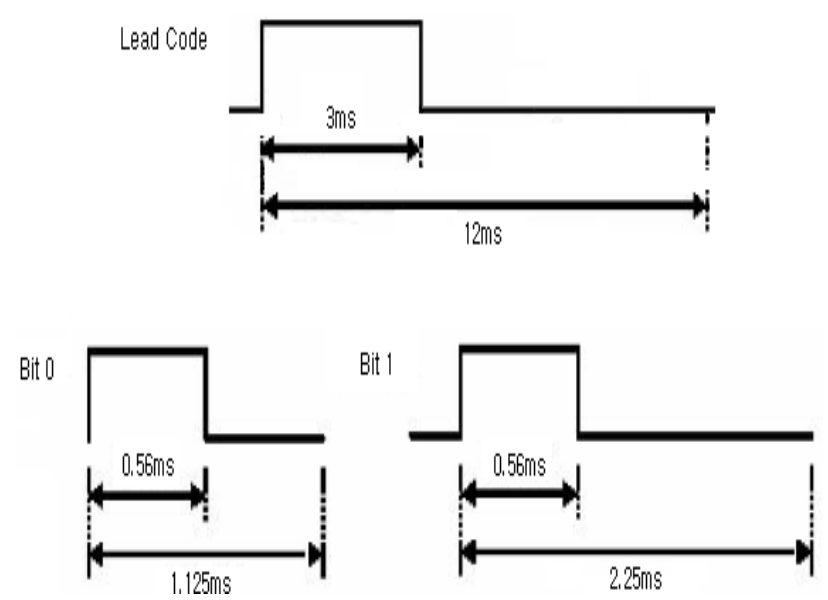

Figure 3. IR Code Structure for the Air-Conditioner

\section{Temperature Measurement}

To acquire room temperature, we have used a contactless thermostat (LM35DZ[5]). The sensor expresses $1{ }^{\circ} \mathrm{C}$ change with $10 \mathrm{mv}$ output. We used the ADC(Analog to Digital Circuit) module provided by the embedded MCU to convert this analog quantity toequivalent digital digits for temperature. With the sensor we were able to describe up to one decimal fixed point accuracy. This temperature value is then sent to the AVRdaemonon the host PC for further processing. When the user connects to the controlling web page from the smart phone, a triggering action to retrieve temperature from the AVRdaemon takes place and the web server provides the temperature to the user, which is acquired through the thermo sensor on the embedded unit.

\section{System Demonstration}

In this system users connect to the web server on the host PC via web browser regardless of the type of mobile terminal they use. Once an event takes place (such as a button click) the web server activates a servlet to handle the request. The servlet sends commands to the AVRdaemon which runs in background to handle various roles of the embedded module. The daemon makes up commands that the embedded module understands andthe embedded module eventually makes up IR signals to be emitted by the LED unit. Hence the air conditioner is being controlled. Three different commands are implemented. They are commands for turningthe air-conditioner on and off, and a command to set the air-conditioner in a certain temperature. Figure 4 shows the power control (power on/off) from the mobile terminal.

Figure 5 depicts the way a user sets the desired temperature. Once the value is set, the intent is sent to AVRdaemon through the servlet.The daemon remembers the value and compares it with the temperature that the embedded module acquires from the sensor. If the room temperature is $2^{\circ} \mathrm{C}$ less than the requested temperature, the daemon requests the embedded module to send out a power-off IR signal.If the room temperature is $2^{\circ} \mathrm{C}$ more than the requested temperature, the daemon orders the embedded module to send out a 
power-on IR signal. If the user requests the power-off from the mobile terminal such automated temperature control is turned off.

The embedded module constantly measures the room temperature. The value is used for the automated temperature setting. The value is also delivered to the user terminal for display so that real time monitoring and control of the air conditioner by the user is possible. The delivering action is triggered by the user: Once the web page is connected by the user, actions for getting current temperature aretriggered in a certain time interval(currently set to 3 seconds). This logic is established by a delay logic implemented in Java Servelet.

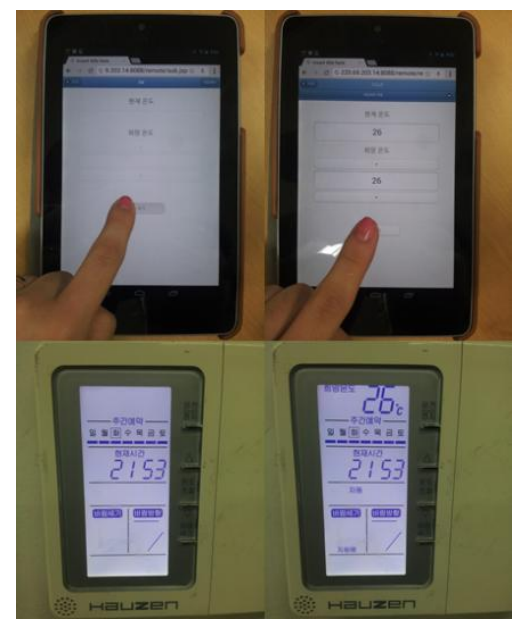

Figure 4. Power Control Demonstration

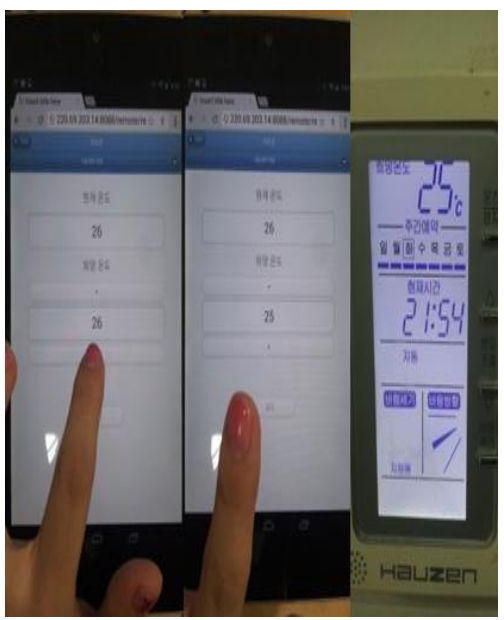

Figure 5. Temperature Control Demonstration

\section{Conclusion}

In this study we have proposed a multi-purpose remote Home Automation model called Whaut. Whaut is a web server/client based remote control model in which an embedded unit is placed to control in-house electric/electronic devices such as appliances. A daemon process is there to monitor and to logically control the embedded unit. Users use a mobile device to 
operate the in-house devices remotely so that the in-house devices controlled anywhere anytime.

We have demonstrated the usefulness of Whaut by implementing a remote air-conditioner controlling system. In this system anembedded unit controls the air-conditionervia IR signal. It also measures the current room temperature so that the values are used to control the roomtemperature automatically. Effectiveness of the system is demonstrated by its actual implementation and usage.

In the future study, we will try implementing many other remote Home Automation applications using Whaut model such as for heaters, TVs, and many other appliances controlled by a remote controller. We are also interested inremotely handling non-remote controller oriented devices such as lights, by wiring a controlling unit that can be instructed by an embedded unit.

\section{Acknowledgments}

This work was supported by the Soonchunhyang University Research Fund.

\section{References}

[1] M. Weiser, "Some Computer Science Problemsin Ubiquitous Computing", Communications of the ACM, vol. 36, no. 7, (1993) July, pp. 75-78.

[2] N. Sriskanthan, F. Tan and A. Karande, "Bluetooth based home automation system", Microprocessors and microsystems, vol. 26, no. 6, (2002) August, pp. 281-289.

[3] C. Otero and R. Larsen, Professional jQuery, Wrox, (2012).

[4] C. D. Knutson and J. M. Brown, IrDA Principles and Protocols, MCL, (2004).

[5] Texas Instruments, (2013) July, LM35 Precision Centigrade Temperature Sensors, http://www.ti.com/lit/ds/symlink/lm35.pdf.
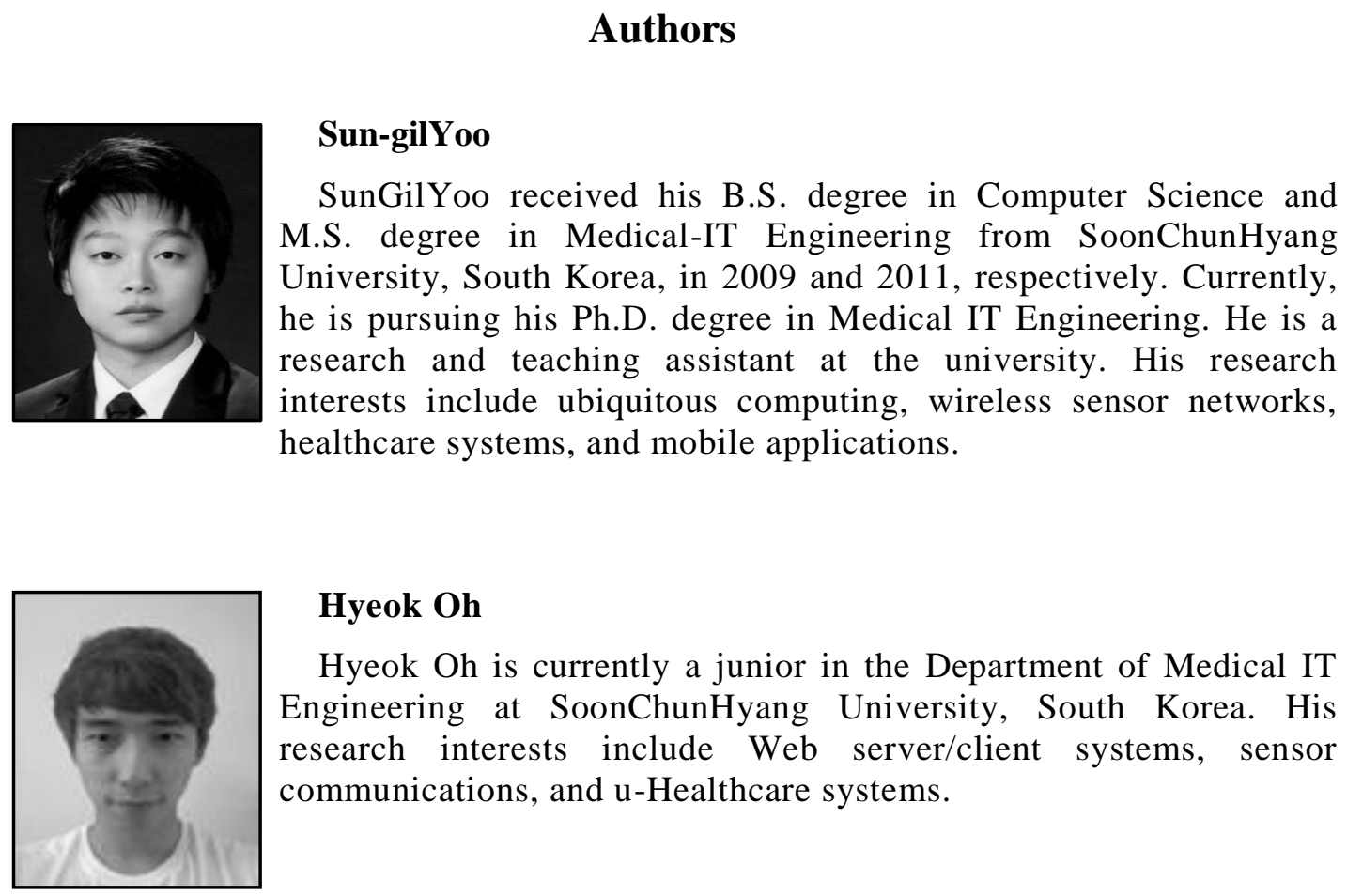

\section{Hyeok Oh}

Hyeok Oh is currently a junior in the Department of Medical IT Engineering at SoonChunHyang University, South Korea. His research interests include Web server/client systems, sensor communications, and u-Healthcare systems. 


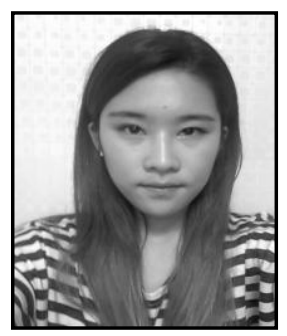

\section{Eunji Kim}

Eunji Kim is currently a junior in the Department of Medical IT Engineering at SoonChunHyang University, South Korea. His research interests include embedded systems, web programming based on JSP and HTML5, and u-Healthcare systems.

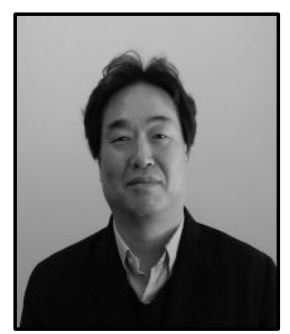

\section{Dongik Oh}

Dong-Ik Oh is currently a professor of Medical IT Engineering at SoonChunHyang University, South Korea. He received his B.S. degree in Computer Science from The City University of New York (College of Staten Island), USA in 1985; M.S. degree in Computer Science from Florida State University, USA in 1989; and Ph.D. degree in Computer Science from Florida State University, USA in 1997. His current area of research includes embedded systems, ubiquitous computing, healthcare systems, and mobile programming.

\section{*Corresponding author: Dongik Oh, Ph.D.}

Department of Medical IT Engineering

College of Medical Sciences, Soonchunhyang University, Asan 336-745, South Korea

E-mail: dohdoh@sch.ac.kr 
International Journal of Control and Automation Vol.7, No.4 (2014) 